“C 2016 IEEE. Personal use of this material is permitted. Permission from IEEE must be obtained for all other uses, in any current or future media, including reprinting/republishing this material for advertising or promotional purposes, creating new collective works, for resale or redistribution to servers or lists, or reuse of any copyrighted component of this work in other works." 


\section{Compact Crossed-Dipole Antennas Loaded With Near-Field Resonant Parasitic Elements}

\author{
Son Xuat Ta, Keekeun Lee, Ikmo Park, and Richard W. \\ Ziolkowski
}

\begin{abstract}
Two compact planar crossed-dipole antennas loaded with near-field resonant parasitic (NFRP) elements are reported. The NFRP and crossed-dipole elements are designed for the desired CP radiation. By placing the NFRP element over the driven element at angles of $0^{\circ}$ and $45^{\circ}$, respectively, dual-band and broadband $\mathrm{CP}$ antennas are realized. Both antennas are $35 \mathrm{~mm} \times 35 \mathrm{~mm} \times 0.508 \mathrm{~mm}\left(0.187 \lambda_{0} \times 0.187 \lambda_{0} \times 0.0027 \lambda_{0}\right.$ at $1.6 \mathrm{GHz})$ in size. The dual-band $\mathrm{CP}$ antenna has a measured $\left|\mathrm{S}_{11}\right|<$ -10-dB bandwidth of $226 \mathrm{MHz}(1.473-1.699 \mathrm{GHz})$ and measured 3-dB axial ratio (AR) bandwidths of $12 \mathrm{MHz}(1.530-1.542 \mathrm{GHz})$ and $35 \mathrm{MHz}(1.580-1.615 \mathrm{GHz})$ with minimum AR CP frequencies of $1.535 \mathrm{GHz}(\mathrm{AR}=0.26 \mathrm{~dB})$ and $1.595 \mathrm{GHz}(\mathrm{AR}=$ $2.08 \mathrm{~dB}$ ), respectively. The broadband $C P$ antenna has a measured $\left|S_{11}\right|<-10-d B$ bandwidth of $218 \mathrm{MHz}(1.491-1.709$ GHz) and a 3-dB AR bandwidth of $145 \mathrm{MHz}(1.490-1.635 \mathrm{GHz})$. These compact antennas yield bi-directional electromagnetic fields with high radiation efficiency across their operational bandwidths.
\end{abstract}

Index Terms-Broadband, circular polarization, crossed dipole, dual-band, near-field resonant parasitic elements

\section{INTRODUCTION}

$\mathrm{T}$ HE design of small antennas has received considerable attention with the expansion of wireless communications in the last several decades [1]. Recently, metamaterial (MTM)-based antennas [2]-[4] have been proposed as a methodology to design small antennas. These are a class of antennas inspired by MTMs to enhance their capability or to achieve novel functions [3]. As an example of a MTM-inspired antenna, near-field resonant parasitic (NFRP) antennas have been widely developed for many applications [2]. The NFRP element is constructed from a single MTM unit cell. When it is properly incorporated with an electrically small driven element, nearly complete impedance matching and high radiation efficiency (RE) are achieved. Depending on the current distribution on the radiating element, the fundamental resonance of an NFRP antenna can be either electric or magnetic. To achieve the conditions for circularly polarized (CP) emissions with a single feed [5], two NFRP elements arranged in an orthogonal configuration must generate two resonances, i.e., one that is tuned to resonate below the desired operating frequency, creating an inductive (or capacitive)

Manuscript received August 12, 2016. This work was supported by the Ajou University research fund and the National Research Foundation of Korea (NRF) grant funded by the Korea government (MEST) (2016R1A2B100932).

Son Xuat Ta, Keekeun Lee, and Ikmo Park (Corresponding Author) are with the Department of Electrical and Computer Engineering, Ajou University, 206 Worldcup-ro, Youngtong-gu, Suwon 16499, Republic of Korea (e-mail: tasonxuat@ajou.ac.kr, keekeun@ajou.ac.kr, ipark@ajou.ac.kr).

Richard W. Ziolkowski is with Department of Electrical and Computer Engineering University of Arizona, 1230 East Speedway Blvd., Tucson, AZ 85721 USA, and also with University of Technology Sydney, Global Big Data Technologies Centre, Ultimo NSW 2000, Australia (e-mail: ziolkowski@ece.arizona.edu). reactance, and one that is tuned to resonate above the desired operating frequency, creating a capacitive (or inductive) reactance. Based on this approach, several electrically small NFRP antennas with CP radiation have been reported [6]-[11]. However, the configurations of these $\mathrm{CP}$ antennas are complicated, and it is difficult to implement them for multi-band operations or to provide a bandwidth enhancement.

A crossed-dipole antenna is a common, modern antenna used to produce CP radiation [12]. Recently, some advances in the design of single-feed crossed-dipole antennas, such as their miniaturization and multi-band operation, were reported [13]-[16]. Miniaturization was realized with the inclusion of a meander line sections and arrowhead-shaped terminations of each dipole arm. To achieve multi-band operation, each dipole arm was divided into multiple branches with different lengths to obtain the requisite set of multiple resonances. However, to realize these multi-frequencies with a small frequency ratio, e.g., to obtain the global positioning system (GPS) L5 (1.17645 $\mathrm{GHz})$ and $\mathrm{L} 2(1.2276 \mathrm{GHz})$ frequencies, one had to face undesired couplings between their crossed multi-branched dipoles [16]. These couplings reduced the RE values and caused a shift in the desired resonance frequency.

In this paper, two related compact crossed-dipole antennas loaded with NFRP elements are investigated. The NFRP element is stacked above the crossed dipoles to realize a planar configuration. By placing the NFRP element over the crossed-dipole element at angles of $0^{\circ}$ and $45^{\circ}$, respectively, dual-band and broadband CP antennas are realized. The promising features of these antennas-planar configuration, compactness, good impedance-matching, good CP radiation, and high RE-were first characterized numerically using the ANSYS High-Frequency Structure Simulator (HFSS) and were then confirmed experimentally.

\section{DUAL-BAND CP CROSSED-DIPOLE NFRP ANTENNA}

\section{A. Antenna Geometry}

Fig. 1 shows the geometry of the dual-band CP antenna. It is composed of an NFRP element, a driven crossed-dipole antenna, and a 50- $\Omega$ coaxial line. These elements are realized with two disks of Rogers RT/Duroid ${ }^{\mathrm{TM}} 5880\left(\varepsilon_{r}=2.2, \mu_{r}=1\right.$, $\tan \delta=0.009$, and $\left.h_{s 1}=h_{s 2}=0.508 \mathrm{~mm}\right)$. The NFRP element was printed on top of the substrate 2 and then stacked over the crossed dipoles without an air gap. The crossed dipoles were designed to be on both sides of substrate 1 and are directly fed by the $50-\Omega$ coaxial line [14]. The crossed dipoles included a pair of vacant-quarter printed rings to produce the $\mathrm{CP}$ radiation. To attain a compact form, a meander line was inserted in each arm of both the driven and the NFRP elements, and each arm was terminated with an arrowhead-shaped trace. To achieve a lower frequency band, the arrowhead-ends of the NFRP element were designed with larger-sizes as compared to the crossed-dipole element. Also, they were designed with different sizes to meet the conditions for CP radiation [5].

The crossed-dipole antenna and its loading with the NFRP element were optimized with a series of HFSS simulations. The optimized final design was matched to the $50-\Omega$ coaxial line without any matching network, and it produced a dual-band CP radiation with a small frequency ratio. Referring to Fig. 1, the 


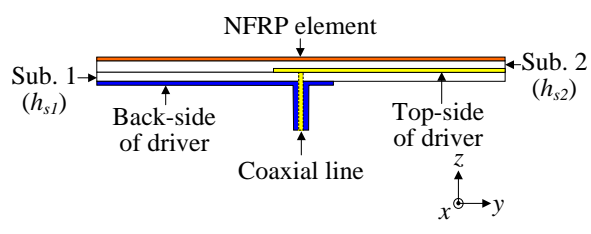

(a)

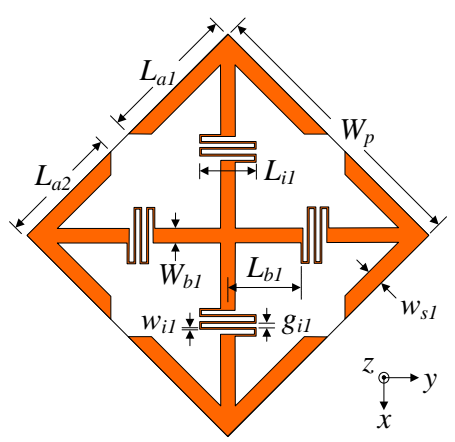

(b)

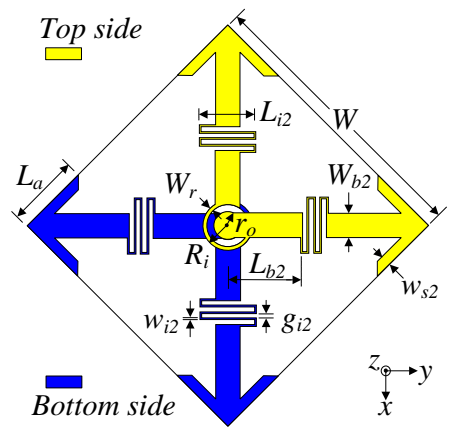

(c)

Fig.1. Geometry of the crossed-dipole NFRP antenna: (a) cross-sectional view, (b) top view of the NFRP element, and (c) top view of the crossed dipoles.

optimized design parameters, all in millimeters, were: $W_{p}=35$, $W_{b 1}=1.8, L_{a 1}=17.3, L_{a 2}=14.5, L_{b 1}=9, w_{i 1}=0.2, g_{i 1}=0.6, L_{i 1}$ $=7, w_{s 1}=2, W=35, R_{b}=3, W_{r}=0.4, r_{o}=1.5, W_{b 2}=3, L_{a}=$ 8.84, $L_{b 2}=9, w_{i 2}=0.2, g_{i 2}=0.6, L_{i 2}=7, w_{s 2}=1.3, h_{s 1}=0.508$, and $h_{\mathrm{s} 2}=0.508$. At $1.6 \mathrm{GHz}$, the size of the antenna is electrically small: $k a=0.83$, where $a$ is the radius of the smallest sphere enclosing the entire antenna and $k=2 \pi / \lambda_{0}, \lambda_{0}$ being the free-space wavelength corresponding to its resonance frequency: $f_{0}$.

\section{B. Antenna Characteristics}

Fig. 2 shows a comparison between the performance characteristics of the crossed-dipole antenna with and without the NFRP element. For a fair comparison, both designs with the same size were optimized to achieve good impedance matching and axial ratio (AR) characteristics at $1.6 \mathrm{GHz}$. Therefore, the design parameters of the antenna without an NFRP element are slightly different from those of the prototype in Fig. 1(c) and are as follows in millimeters: $W=35, R_{b}=3.2, W_{r}=0.5, r_{o}=$ 2.2, $W_{b 2}=3.2, L_{b 2}=10, w_{i 2}=0.2, g_{i 2}=0.6, L_{i 2}=7, L_{a}=10.25$, $w_{s 2}=2$, and $h_{s 1}=0.508$. In this configuration, the antenna has a single-band of CP operation with a $\left|\mathrm{S}_{11}\right|<-10$-dB bandwidth of $206 \mathrm{MHz}(1.552-1.758 \mathrm{GH})$ with two resonances at $1.587 \mathrm{GHz}$ and $1.695 \mathrm{GHz}$. It has a 3-dB AR bandwidth of $42 \mathrm{MHz}$

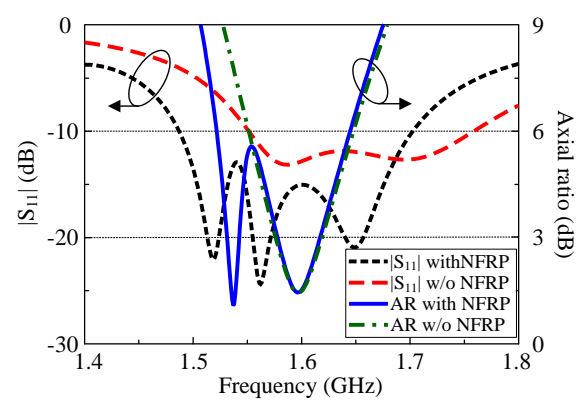

Fig. 2. Simulated $\left|S_{11}\right|$ and AR values as functions of the source frequency for the planar crossed-dipole antenna with and without the NFRP element.

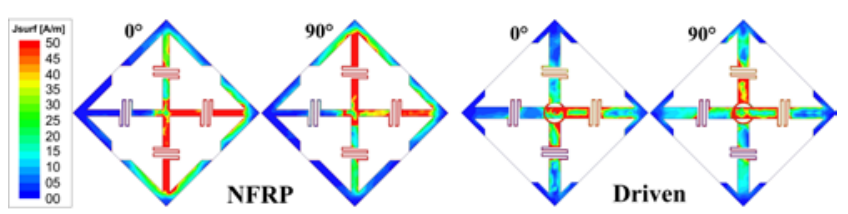

(a)

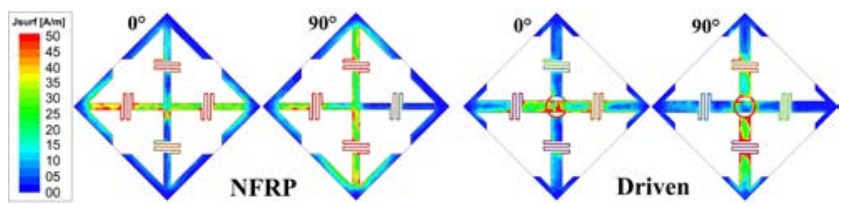

(b)

Fig. 3. Current distributions on the NFRP and crossed-dipole elements of the dual-band CP antenna at (a) 1.537 and (b) $1.597 \mathrm{GHz}$.

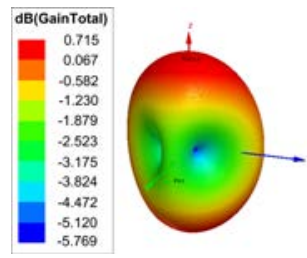

(a)

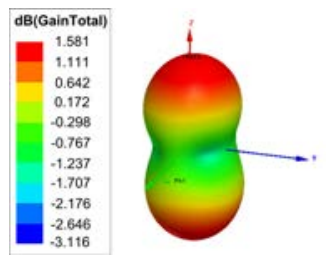

(b)
Fig. 4. Simulated 3D total gain patterns of the dual-band CP antenna at (a) $1.537 \mathrm{GHz}$ and (b) $1.597 \mathrm{GHz}$.

(1.576-1.618 GHz) with its $\mathrm{CP}$ frequency band centered at $1.597 \mathrm{GHz}(\mathrm{AR}=1.44 \mathrm{~dB})$.

In comparison, the crossed-dipole antenna loaded with the NFRP element yields a larger $\left|S_{11}\right|<-10$-dB bandwidth of 215 $\mathrm{MHz}$ (1.487-1.702 GHz) with three resonances at 1.519, 1.562, and $1.649 \mathrm{GHz}$. It has 3-dB AR bandwidths of $10 \mathrm{MHz}$ (1.532-1.542 GHz) and $39 \mathrm{MHz}(1.578-1.617 \mathrm{GHz})$ with two $\mathrm{CP}$ frequency bands centered at $1.537 \mathrm{GHz}(\mathrm{AR}=1.07 \mathrm{~dB})$ and $1.597 \mathrm{GHz}(\mathrm{AR}=1.45 \mathrm{~dB})$. These results indicate that due to the presence of the NFRP element, the antenna becomes a dual-band $\mathrm{CP}$ radiator.

To illustrate the CP behavior of this dual-band CP antenna, the current distributions on the NFRP element and crossed dipoles were computed for different phase angles. The current distributions for the two minimum points at 1.537 and 1.597 $\mathrm{GHz}$ in the AR profile of the design are given in Fig. 3. As shown in Fig. 3(a), the horizontal arms of both the NFRP and driven crossed-dipole elements are resonant at $1.537 \mathrm{GHz}$ for the $0^{\circ}$ phase angle, whereas only their vertical arms are resonant for the $90^{\circ}$ phase angle. In addition, it was observed at $1.537 \mathrm{GHz}$ that the currents on the NFRP element were stronger 
compared with those on the crossed dipoles. These results indicate that the lower CP frequency band is caused by the NFRP element. Additionally, the current distributions at 1.537 $\mathrm{GHz}$ were not very symmetric on both elements. This behavior occurs because the crossed dipoles are placed on different sides of the substrate 1, i.e., the near-field coupling between the NFRP element and the dipole arms on the top side (spacing of $h_{s 2}$ ) is stronger than that between the NFRP element and the dipole arms on the bottom side (spacing of $h_{s 1}+h_{\mathrm{s} 2}$ ). As shown in Fig. 3(b), the horizontal arms of both the NFRP and the crossed-dipole elements resonate at $1.597 \mathrm{GHz}$ for the $0^{\circ}$ phase angle, whereas only their vertical arms resonate for the $90^{\circ}$ phase angle. It also indicates that the currents on the crossed-dipole element were stronger than those on the NFRP element. This proves that the upper CP frequency band is caused by the driven element.

Similar to the single-feed crossed-dipole antennas [14]-[16], the vacant-quarter printed rings were used in the optimized design. They equally distribute the input signal from the coaxial line between the dipole arms on the bottom and top sides of substrate 1 . Therefore, the current distributions on the crossed dipole at $1.597 \mathrm{GHz}$ are relatively symmetric at both phase angles.

It is well known that the current distribution on the antenna determines its radiation profile. This is confirmed in Fig. 4, which shows the simulated 3D total gain patterns of the optimized antenna at 1.537 and $1.597 \mathrm{GHz}$. According to the current distributions shown in Figs. 3 and 4, the $1.537 \mathrm{GHz}$ gain pattern was slightly asymmetric, while the $1.597 \mathrm{GHz}$ gain pattern was quite symmetric. Although there is a slight difference in the gain pattern profile, the antenna basically radiated a bi-directional electromagnetic field with high RE values at both frequencies. The HFSS simulations predicted an $\mathrm{RE}$ of $72 \%$, and both the broadside and backside gains predicted were $\sim 0.7 \mathrm{dBic}$ at $1.537 \mathrm{GHz}$. At $1.597 \mathrm{GHz}$, a RE value of $89 \%$ and broadside and backside gains, respectively, of 1.5 and $1.53 \mathrm{dBic}$ were predicted.

\section{Measurements}

The dual-band CP antenna was fabricated and measured to verify the simulated performance characteristics. The crossed-dipole and NFRP elements were printed on the Rogers RT/Duroid ${ }^{\mathrm{TM}} 5880$ substrates with standard wet-etching technology. Fig. 5 shows the fabricated prototype, which has an overall size of $35 \mathrm{~mm} \times 35 \mathrm{~mm} \times 1.016 \mathrm{~mm}(\mathrm{ka}=0.83$ at 1.6 GHz). Fig. 6 shows the comparison between the measured and simulated $\left|S_{11}\right|$ and AR values for the fabricated prototype. The measurement results showed a $\left|\mathrm{S}_{11}\right|<-10-\mathrm{dB}$ bandwidth of 226 MHz (1.473-1.699 GHz) in contrast to the predicted value: 215 MHz. The measured results gave AR $<3.0 \mathrm{~dB}$ bandwidths of $12 \mathrm{MHz}(1.530-1.542 \mathrm{GHz})$ and $35 \mathrm{MHz}(1.580-1.615 \mathrm{GHz})$, in contrast to the corresponding simulated 3-dB AR bandwidths: 10 and $39 \mathrm{MHz}$, respectively. The measurements yielded two minimum $\mathrm{AR}$ points at $1.535 \mathrm{GHz}(\mathrm{AR}=0.26 \mathrm{~dB}$ ) and $1.595 \mathrm{GHz}(\mathrm{AR}=2.08 \mathrm{~dB})$, which were only slightly shifted from their simulated values of 1.537 and $1.597 \mathrm{GHz}$.

Fig. 7 shows the gain patterns of the dual-band CP antenna for the lower and upper CP frequency bands. Both the simulations and measurements show that the antenna radiates a bi-directional electromagnetic wave, i.e., the broadside

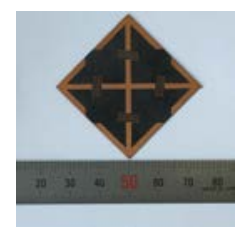

(a)

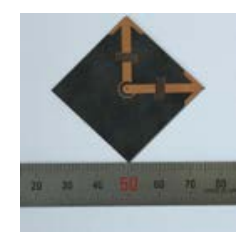

(b)

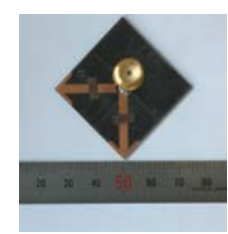

(c)
Fig. 5. Fabricated prototype of the dual-band CP antenna: (a) top view of the NFRP element, (b) top view of the crossed-dipole element, and (c) bottom-view showing the cross-dipole element and the coaxial feed.

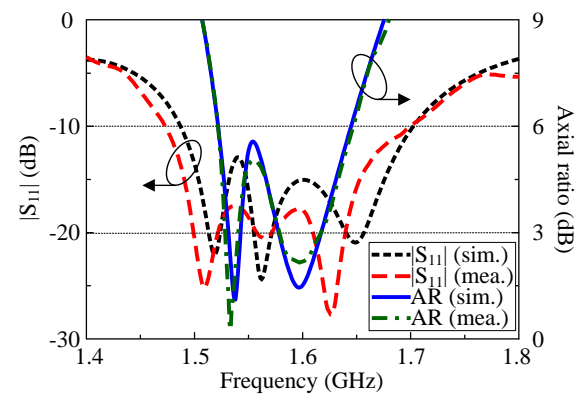

Fig. 6. Simulated and measured $\left|S_{11}\right|$ and AR values as functions of the source frequency for the fabricated dual-band CP antenna.
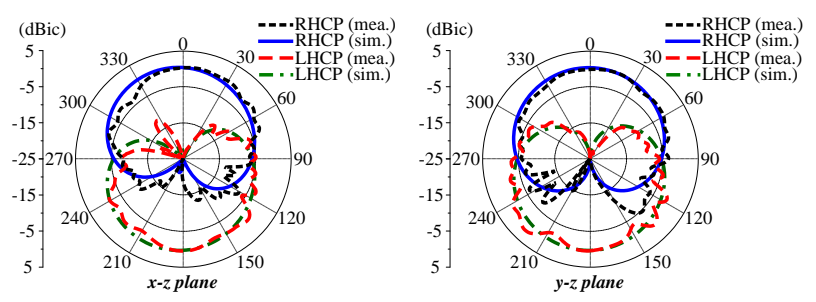

(a)

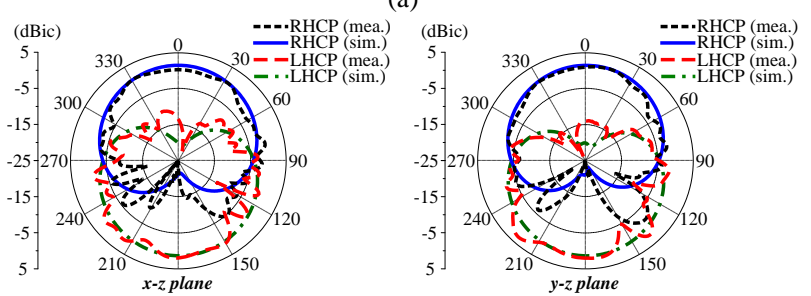

(b)

Fig. 7. Simulated and measured total gain patterns of the fabricated dual-band CP antenna at its minimum AR frequencies. The simulation results are at (a) 1.537 and (b) $1.597 \mathrm{GHz}$, whereas the measurement results are at (a) 1.535 and (b) $1.595 \mathrm{GHz}$.

radiation is right-hand circularly polarized (RHCP), while the backside radiation is left-hand circularly polarized (LHCP). At the lower band, the measured broadside and backside gain values were 0.6 and $0.8 \mathrm{dBic}$, respectively. At the upper band, the measured broadside and backside gains were 1.4 and 1.52 $\mathrm{dBic}$, respectively. There were some ripples in the measured results, which could be attributed to the effects of the foam rack and the tapes employed in the pattern-measurement setup. Additionally, the antenna yielded measured RE and gain values greater than $70 \%$ and $0.5 \mathrm{dBic}$ within its operational bandwidth, respectively.

\section{Crossed-Dipole Antenna LoAded With Rotated NFRP ELEMENT}

The crossed-dipole antenna loaded with an NFRP element was first characterized for different rotation angles $(\alpha)$ of the 
parasitic element with respect to the driven element [Fig. 8(a)], i.e., $\alpha=0^{\circ}, 45^{\circ}$, and $90^{\circ}$. The driven and parasitic elements of all these configurations have the same basic structure as the one shown in Fig. 1. All elements were implemented on Rogers $\mathrm{RT} /$ Duroid $^{\mathrm{TM}} 5880$ substrates. When $\alpha=0^{\circ}$, the design produced the dual-band CP antenna described in the previous section. For a fair comparison, the configurations with $\alpha=45^{\circ}$ and $90^{\circ}$ were optimized with the same electrical size: $k a=0.83$, and good impedance matching at $1.6 \mathrm{GHz}$. Consequently, their design parameters were slightly different from those of the $\alpha=$ $0^{\circ}$ configuration. Referring to Fig. 1 , the design parameters of the $\alpha=45^{\circ}$ configuration in millimeters were: $W_{p}=35, W_{b}=$ 3.2, $L_{a 1}=16.05, L_{a 2}=13.22, L_{b 1}=10.5, w_{i 1}=0.2, g_{i 1}=0.6, L_{i 1}=$ $6, w_{s 1}=2, W=35, R_{b}=3.4, w_{r}=0.3, L_{a}=8.55, L_{b 2}=11, w_{i 2}=$ $0.2, g_{i 2}=0.6, L_{i 2}=6, w_{s 2}=1.4, h_{s 1}=0.508$, and $h_{s 2}=0.508$. For the $\alpha=90^{\circ}$ configuration, the design parameters in millimeters were: $W_{p}=35, W_{b 1}=1.8, L_{a 1}=17.3, L_{a 2}=14.5, L_{b 1}=9$, $w_{i 1}=$ $0.2, g_{i 1}=0.6, L_{i 1}=8, w_{s 1}=2, W=35, R_{b}=3, W_{r}=0.4, r_{o}=1.5$, $W_{b 2}=3, L_{a}=8.84, L_{b 2}=9, w_{i 2}=0.2, g_{i 2}=0.6, L_{i 2}=8, w_{\mathrm{s} 2}=1.3$, $h_{s 1}=0.508$, and $h_{s 2}=0.508$. The performance characteristics of the antennas with $\alpha=0^{\circ}, 45^{\circ}$, and $90^{\circ}$ were characterized with HFSS; these results are given in Figs. 8(b) $-8(d)$.

It is well known that the overall impedance behavior of the NFRP antenna is controlled by the self-reactances of the driven and NFRP elements and their relative arrangement [2]. Accordingly, the input impedance of the crossed-dipole antenna loaded with an NFRP element is significantly influenced by different rotation angles $(\alpha)$ of the parasitic element. This is observed in Fig. 8(b), which shows the simulated input impedances of the three configurations. When $\alpha=0^{\circ}$, the arms and arrowhead-shaped ends of NFRP element overlapped with those of the crossed dipole. These overlappings result in the maximum capacitance of the antenna system and the design has a negative reactance [Fig. 8(b)]. As described in Section II, the case with $\alpha=0^{\circ}$ was optimized to achieve a broad impedance matching bandwidth. This was due to a proper coupling between its elements, i.e., the horizontal dipole of the driven element, which was the shorter dipole, was directly coupled with the smaller-ending arms of the NFRP element, whereas the vertical dipole, which was longer because of the addition of the ring, was directly coupled with the larger-ending arms of the NFRP element. As $\alpha$ increased up to $45^{\circ}$, the capacitance of the antenna system decreased because of the reduction of the overlapping between the arms and arrowhead ends.

On the other hand, the reduction of the overlapping between their meander-lines increased the inductances of both the driven and parasitic elements; therefore, the inductance of the antenna system increased significantly. As a result, the configuration with $\alpha=45^{\circ}$ has the minimum capacitance and the maximum inductance. In order to compensate for these reactances, several changes, such as wider arm-widths ( $W_{b 1}$ and $W_{b 2}$ ), shorter meander-lines $\left(L_{i 1}\right.$ and $\left.L_{i 2}\right)$, and a larger radius $\left(r_{o}\right)$ of the feeding disk, were incorporated into the driven and NFRP elements of the $\alpha=45^{\circ}$ configuration to obtain good impedance matching at the desired frequency band. As shown in Fig. 8(c), the crossed-dipole antenna loaded with the $45^{\circ}$-rotated NFRP element yields a bandwidth of $180 \mathrm{MHz}$ $(1.525-1.705 \mathrm{GHz})$ for $\left|\mathrm{S}_{11}\right|<-10 \mathrm{~dB}$. When $\alpha$ increased from $45^{\circ}$ to $90^{\circ}$, the overlap between the crossed-dipole and NFRP

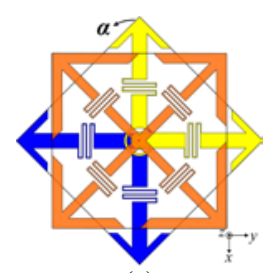

(a)

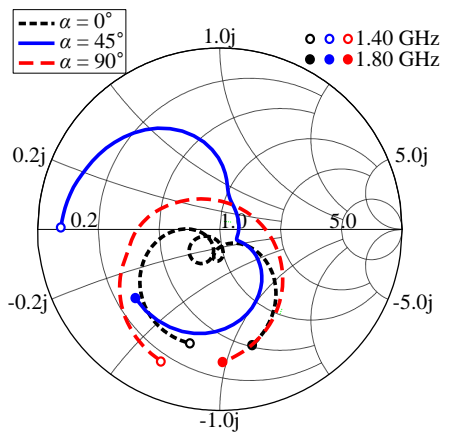

(b)

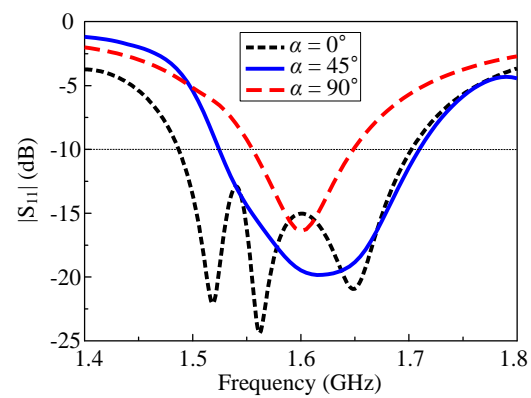

(c)

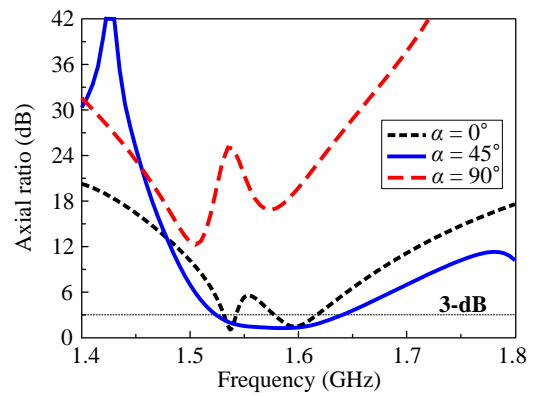

(d)

Fig. 8. The performance characteristics of the driven crossed-dipole antennas loaded with the rotated NFRP element for different rotation angles $\alpha$. (a) Geometry, and (b) the simulated input impedance, and the (c) $\left|\mathrm{S}_{11}\right|$ and (d) AR values as functions of the source frequency.

elements, including their arms, meander-lines, and arrowhead ends, increased. Accordingly, the capacitance of the antenna system increased, whereas its inductance decreased. These variations are the opposite of those observed when $\alpha$ increases from $0^{\circ}$ to $45^{\circ}$.

It was predicted that the behavior of the input impedance for the $\alpha=90^{\circ}$ configuration could be similar to that of the $\alpha=0^{\circ}$ case. However, the coupling between the NFRP and crossed-dipole elements of the case with $\alpha=90^{\circ}$ was different from the $\alpha=0^{\circ}$ design, i.e., the horizontal dipole of the driven element, which was the shorter dipole, was directly coupled with the larger-ending arms of the NFRP element. In contrast, its vertical dipole, which was longer, was directly coupled with the smaller-ending arms of the NFRP element. These couplings 
deteriorated the impedance matching bandwidth of the antenna. As shown in Fig. 8(c), its $\left|\mathrm{S}_{11}\right|<-10 \mathrm{~dB}$ bandwidth is $1.560-1.645 \mathrm{GHz}$ with one resonance at $1.60 \mathrm{GHz}\left(\left|\mathrm{S}_{11}\right|=\right.$ $-16.4 \mathrm{~dB})$.

Fig. 8(d) shows the AR values of the crossed-dipole antenna loaded with an NFRP element for different rotation angles $(\alpha)$. When $\alpha=0^{\circ}$, the proper coupling between the NFRP and crossed-dipole elements produced a good RHCP radiation at broadside ( $+\mathrm{z}$ direction) for both bands. The AR characteristics of this design were described in Section II. With the $45^{\circ}$-rotated NFRP element, the antenna yielded a broadband CP radiation at the frequency band of interest. It had a 3-dB AR bandwidth of $115 \mathrm{MHz}(1.525-1.640 \mathrm{GHz})$ with two CP frequency bands centered at $1.55 \mathrm{GHz}(\mathrm{AR}=1.5 \mathrm{~dB})$ and $1.60 \mathrm{GHz}(\mathrm{AR}=1.3$ $\mathrm{dB}$ ). The radiation mechanism of this design is discussed in the next section.

As mentioned above, in the case with $\alpha=90^{\circ}$, the shorter dipole of the driven element was directly coupled with the larger-ending arms of the NFRP element, whereas the longer dipole of the driven element was directly coupled with the smaller-ending arms of the NFRP element. These couplings degraded one of the two resonances caused by the crossed-dipole itself [Fig. 8(c)]; and consequently, they altered the conditions for $\mathrm{CP}$ radiation of the driven element. As shown in Fig. 8(d), the design with $\alpha=90^{\circ}$ was nearly linear polarized, i.e., $\mathrm{AR}=19.7 \mathrm{~dB}$ at $1.6 \mathrm{GHz}$.

\section{BROADBAND CP CROSSED-Dipole NFRP ANTENNA}

\section{A. Antenna Characteristics}

As shown in Fig. 8(d), the crossed-dipole antenna loaded with the $45^{\circ}$-rotated NFRP element yields a broadband CP radiation. For a better understanding of its radiation mechanism, the current distributions on the NFRP and crossed-dipole elements were computed for different phase angles. The current distributions for the two minimum points at 1.55 and $1.60 \mathrm{GHz}$ in the AR profile of the design are given in Fig. 9. As shown in Fig. 9(a), the currents on the NFRP element at $1.55 \mathrm{GHz}$ are stronger compared with the currents on the crossed-dipole element for both phase angles. These results indicate that the lower CP band was caused by the NFRP element. In addition, it is observed that the NFRP element is excited by the horizontal and vertical arms of the crossed-dipole element at the $0^{\circ}$ and $90^{\circ}$ phase angles, respectively. This indicates that the design produces a RHCP fields at broadside. As shown in Fig. 9(b), the currents on the crossed-dipole element at $1.60 \mathrm{GHz}$ were stronger when compared with the currents on the NFRP element, implying that the upper CP frequency band is caused by the driven element. Furthermore, the current distributions indicate that the antenna also produces RHCP fields at broadside, i.e., the horizontal arms of the crossed-dipole elements resonate at 1.60 $\mathrm{GHz}$ for the $0^{\circ}$ phase angle, whereas only its vertical arms resonate for the $90^{\circ}$ phase angle.

Unlike the dual-band CP antenna presented in Section II, the current distributions on the crossed-dipole antenna loaded with the $45^{\circ}$-rotated NFRP element are quite symmetric at both CP bands, as illustrated in Fig. 9. This behavior is due to the rotation of the NFRP element, which overcomes the different near-field couplings between the parasitic element and the
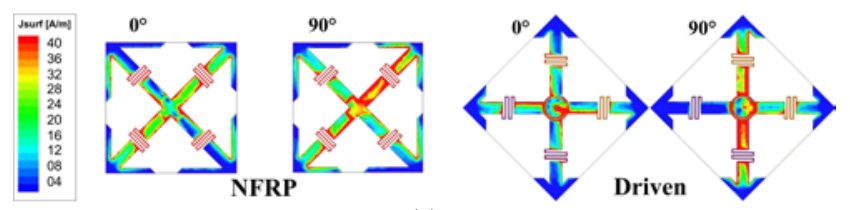

(a)
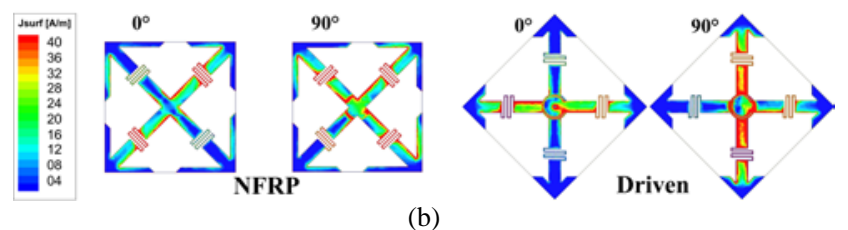

(b)

Fig. 9. Current distributions on the NFRP and crossed-dipole elements of the broadband CP antenna at (a) $1.55 \mathrm{GHz}$ and (b) $1.60 \mathrm{GHz}$.

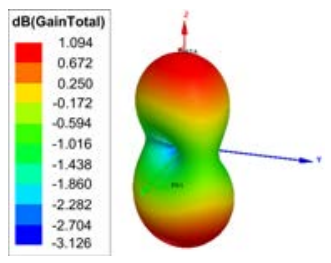

(a)

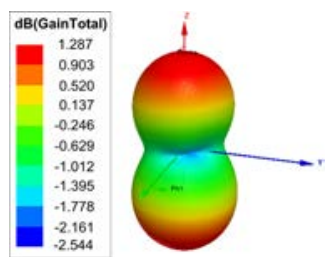

(b)
Fig. 10. Simulated 3D total gain patterns of the broadband CP antenna at (a) $1.55 \mathrm{GHz}$ and (b) $1.60 \mathrm{GHz}$.

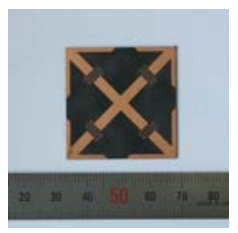

(a)

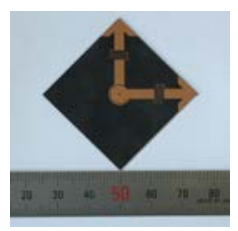

(b)

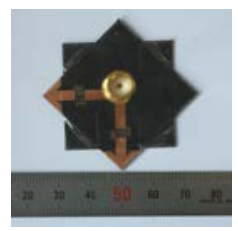

(c)
Fig. 11. Fabricated prototype of the broadband CP antenna. (a) Top view of the NFRP element, (b) top view of the crossed-dipole element, and (c) bottom-view showing the cross-dipole element and the coaxial feed.

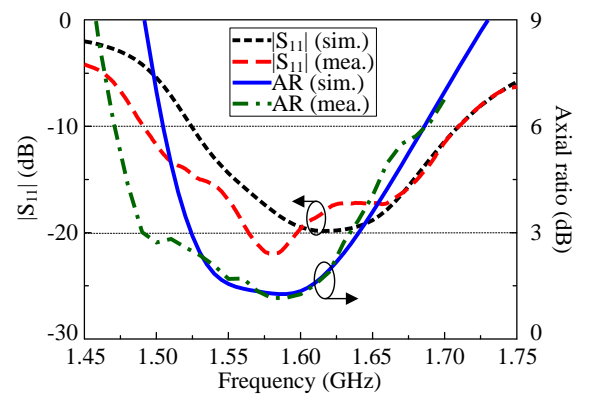

Fig. 12. Simulated and measured $\left|\mathrm{S}_{11}\right|$ and (b) AR values as functions of the source frequency for the fabricated broadband CP antenna.

dipole arms of the driven element on the top and bottom sides of substrate 1 . These current distributions indicate that the antenna has a symmetric radiation profile at both CP bands. This is confirmed in Fig. 10, which shows the simulated 3D total-gain pattern of the crossed-dipole antenna loaded with the $45^{\circ}$-rotated NFRP element at $1.55 \mathrm{GHz}$ and at $1.60 \mathrm{GHz}$. Moreover, the HFSS simulations predict a RE of $79.5 \%$ with broadside and backside gains of 0.82 and $1.04 \mathrm{dBic}$ at $1.55 \mathrm{GHz}$, respectively, and a RE of $85.8 \%$ with broadside and backside gain of 1.13 and $1.26 \mathrm{dBic}$, respectively, at $1.60 \mathrm{GHz}$. 

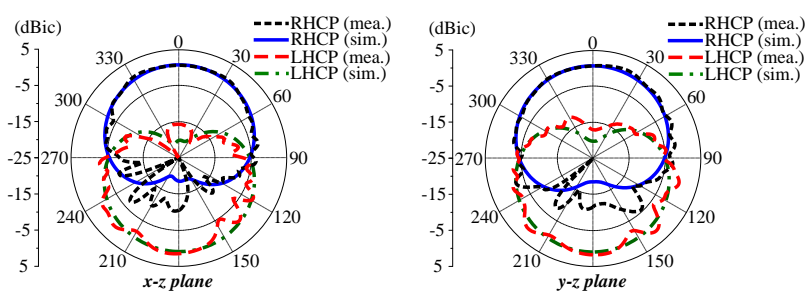

(a)
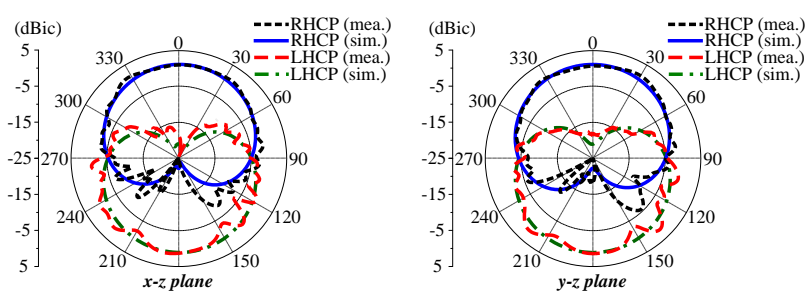

(b)

Fig. 13. Simulated and measured total gain patterns of the fabricated broadband $\mathrm{CP}$ antenna at the two minimum points in its AR profile: (a) 1.55 and (b) $1.60 \mathrm{GHz}$.

\section{B. Measurements}

The crossed-dipole antenna loaded with the $45^{\circ}$-rotated NFRP element was also fabricated and measured. Fig. 11 shows a sample of the fabricated prototype. Both elements again had an overall size of $35 \mathrm{~mm} \times 35 \mathrm{~mm} \times 0.508 \mathrm{~mm}$. Consequently, the antenna size was maintained at $k a=0.83$ at $1.6 \mathrm{GHz}$. Fig. 12 shows the measured and simulated $\left|S_{11}\right|$ and AR values for the fabricated prototype. It is observed that the measurements agreed rather closely with the HFSS predictions. The measured bandwidth for $\left|S_{11}\right|<-10 \mathrm{~dB}$ is $218 \mathrm{MHz}$ (1.491-1.709 GHz), whereas the simulated bandwidth was $180 \mathrm{MHz}$ (1.525-1.705 GHz). The measured 3-dB AR bandwidth is $145 \mathrm{MHz}(1.490-1.635 \mathrm{GHz})$, whereas its simulated value was $115 \mathrm{MHz}(1.525-1.640 \mathrm{GHz})$.

Fig. 13 shows the gain patterns of the fabricated broadband $\mathrm{CP}$ antenna at $1.55 \mathrm{GHz}$ and $1.60 \mathrm{GHz}$. Similar to the dual-band CP antenna, the broadband CP antenna radiated a bi-directional electromagnetic wave, i.e., the broadside radiation was RHCP, while the backside radiation was LHCP. At $1.55 \mathrm{GHz}$, the measured broadside and backside gain values were 1.05 and $1.24 \mathrm{dBic}$, respectively. At $1.60 \mathrm{GHz}$, the measured broadside and backside gains were 1.01 and $1.30 \mathrm{dBic}$, respectively. Similar to the case of the dual-band CP antenna, the measurement results have some ripples in the back-radiation patterns. These ripples are attributed to the effects of the foam rack and the tapes employed in the pattern-measurement setup. Additionally, the antenna yielded measured RE and gain values of greater than $75 \%$ and $1.0 \mathrm{dBic}$ within its operational bandwidth, respectively.

\section{CONCLUSION}

Two compact single-feed crossed-dipole NFRP CP antennas were presented. The parasitic and driven elements were optimized to achieve the desired CP radiation. By placing the NFRP element over the crossed-dipole element with an angle of $0^{\circ}$ and $45^{\circ}$, dual-band and broadband CP antennas, respectively, were realized. Their operating mechanisms were explained with details about their current distributions. These antennas were fabricated with the same size of $k a=0.83$ at $1.6 \mathrm{GHz}$ and measured. Both antennas yielded bi-directional electromagnetic fields and exhibited high RE values across their useful operational bandwidths. The measured and simulated performance characteristics were in good agreement. In comparison to existing multiband CP NFRP antennas, the proposed dual-band CP design has a simpler configuration and is easier to fabricate. As compared to previously reported electrically-small CP antennas with a single-feed, the proposed broadband CP design has a significantly broader AR bandwidth. Because of their planar configuration, electrically small size, good impedance matching, and dual-band/ broadband CP radiation features, the presented crossed-dipole NFRP antennas can be widely used in global navigation satellite systems.

\section{REFERENCES}

[1] H. Wong, K. Luk, C. Chan, Q. Xue, K. So, and H. Lai, "Small antennas in wireless communications,” Proc. IEEE, vol. 100, no. 7, pp. 2109-2121, Jul. 2012.

[2] R. W. Ziolkowski, P. Jin, and C. Lin, "Metamaterial-inspired engineering of antennas," Proc. IEEE, vol. 99, no. 10, pp. 1720-1731, Oct. 2011.

[3] Y. Dong and T. Itoh, "Metamaterial-based antennas," Proc. IEEE, vol. 100, no. 7, pp. 2271-2285, Jul. 2012.

[4] E. J. Rothwell and R. O. Ouedraogo, "Antenna miniaturization: definitions, concepts, and a review with emphasis on metamaterials," $J$. Electromagn. Waves Appl., vol. 28, no. 17, pp. 2089-2123, 2014.

[5] M. F. Bolster, "A new type of circular polarizer using crossed dipoles," IRE Trans. Microw. Theory Tech., vol. 9, no. 5, pp. 385-388, 1961.

[6] C. Lin, P. Jin, and R. W. Ziolkowski, "Multi-functional, magnetically-coupled, electrically small, near-field resonant parasitic wire antennas," IEEE Trans. Antennas Propag., vol. 59, no. 3, pp. 714-724, Mar. 2011.

[7] P. Jin and R. W. Ziolkowski, "Multi-frequency, linear and circular polarized, metamaterial-inspired, near-field resonant parasitic antennas," IEEE Trans. Antennas Propag., vol. 59, no. 5, pp. 1446-1459, May. 2011.

[8] P. Jin, C. Lin, and R. W. Ziolkowski, "Multifunctional, electrically small, planar near-field resonant parasitic antennas," IEEE Antennas Wireless Propag. Lett., vol. 11, pp. 200-204, 2012.

[9] P. Jin and R. W. Ziolkowski, "High directivity, electrically small, low-profile, near-field resonant parasitic antennas," IEEE Antennas Wireless Propag. Lett., vol. 11, pp. 305-309, 2012.

[10] L. Sun, B. Du, and B. Sun, "Inductively loaded and magnetically coupled small antenna with circular polarization,” J. Electromagn. Waves Appl., vol. 27, no. 5, pp. 539 - 543, 2013.

[11] M. Tang and R. W. Ziolkowski, "Frequency-agile, efficient, circularly polarized, near-field resonant antenna: designs and measurements," IEEE Trans. Antennas Propag., vol. 63, no. 11, pp. 5203-5209, Nov. 2015.

[12] S. X. Ta, I. Park, and R. W. Ziolkowski, "Crossed dipole antennas: a review,” IEEE Antennas Propagat. Mag., vol. 57, no. 5, pp. 107-122, Oct. 2015.

[13] H. H. Tran, S. X. Ta, and I. Park, "A compact circularly polarized crossed-dipole antenna for an RFID tag," IEEE Antennas Wireless Propag. Lett., vol. 14, pp. 674-677, 2015.

[14] S. X. Ta, H. Choo, and I. Park, "Planar, lightweight, circularly polarized crossed dipole antenna for handheld UHF RFID reader," Microwave Opt. Technol. Lett., vol. 55, no. 8, pp. 1874-1878, Aug. 2013.

[15] S. X. Ta, I. Park, and R. W. Ziolkowski, "Dual-band wide-beam crossed asymmetric dipole antenna for GPS application,” Electron. Lett., vol. 48, no. 25, pp.1580-1581, Dec. 2012.

[16] S. X. Ta, H. Choo, I. Park, and R. W. Ziolkowski, "Multi-band, wide-beam, circularly polarized, crossed, asymmetrically barbed dipole antennas for GPS applications,” IEEE Trans. Antennas Propag., vol. 61, no. 11, pp. 5771-5775, Nov. 2013. 